\title{
Accretion dynamics and disk evolution in NGC 2264: a study based on CoRoT` photometric observations ${ }^{\star \star}$
}

\author{
S. H. P. Alencar ${ }^{1}$, P. S. Teixeira ${ }^{2}$, M. M. Guimarães ${ }^{1,3}$, P. T. McGinnis ${ }^{1}$, J. F. Gameiro ${ }^{4}$,

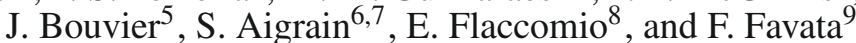 \\ 1 Departamento de Física - ICEx - UFMG, Av. Antônio Carlos, 6627, 30270-901 Belo Horizonte, MG, Brazil \\ e-mail: silvia@fisica.ufmg.br \\ 2 ESO, Karl-Schwarzschild-Strasse 2, 85748 Garching bei München, Germany \\ 3 UFSJ - Campus Alto Paraopeba - Rodovia MG 443, KM 7, 36420-000 Ouro Branco, MG, Brazil \\ 4 Centro de Astrofisica da Universidade do Porto, Rua das Estrelas, 4150 Porto, Portugal \\ 5 Laboratoire d'Astrophysique, Observatoire de Grenoble, BP 53, 38041 Grenoble Cedex 9, France \\ ${ }^{6}$ School of Physics, University of Exeter, Exeter, EX4 4QL, UK \\ 7 Astrophysics, University of Oxford, Denys Wilkinson Building, Oxford, OX4 1DQ, UK \\ 8 INAF - Osservatorio Astronomico di Palermo, Piazza del Parlamento 1, 90134 Palermo, Italy \\ 9 European Space Agency, 8-10 rue Mario Nikis, 75015 Paris, France \\ Received 2 February 2010 / Accepted 19 May 2010
}

ABSTRACT

\begin{abstract}
Context. The young cluster NGC 2264 was observed with the CoRoT satellite for 23 days uninterruptedly in March 2008 with unprecedented photometric accuracy. We present the first results of our analysis of the accreting population belonging to the cluster as observed by CoRoT.

Aims. We search for possible light curve variability of the same nature as that observed in the classical T Tauri star AA Tau, which was attributed to a magnetically controlled inner disk warp. The inner warp dynamics is supposed to be directly associated with the interaction between the stellar magnetic field and the inner disk region.

Methods. We analyzed the CoRoT light curves of 83 previously known classical T Tauri stars that belong to NGC 2264 classifying them according to their light-curve morphology. We also studied the CoRoT light-curve morphology as a function of a Spitzer-based classification of the star-disk systems.

Results. The classification derived on the basis of the CoRoT light-curve morphology agrees very well with the Spitzer IRAC-based classification of the systems. The percentage of AA Tau-like light curves decreases as the inner disk dissipates, from $40 \% \pm 10 \%$ in systems with thick inner disks to $36 \% \pm 16 \%$ in systems with anemic disks and zero in naked photosphere systems. Indeed, $91 \% \pm 29 \%$ of the CTTS with naked photospheres exhibit pure spot-like variability, while only $18 \% \pm 7 \%$ of the thick disk systems do so, presumably those seen at low inclination and thus free of variable obscuration.

Conclusions. AA Tau-like light curves are found to be fairly common, with a frequency of at least $\sim 30$ to $40 \%$ in young stars with inner dusty disks. The temporal evolution of the light curves indicates that the structure of the inner disk warp, located close to the corotation radius and responsible for the obscuration episodes, varies over a timescale of a few ( $\sim 1-3)$ rotational periods. This probably reflects the highly dynamical nature of the star-disk magnetospheric interaction.
\end{abstract}

Key words. accretion, accretion disks - techniques: photometric - stars: pre-main sequence

\section{Introduction}

$\mathrm{T}$ Tauri stars are young, optically visible, low-mass stars still contracting toward the main sequence. They have strong magnetic fields $(\sim 2 \mathrm{kG})$ and are X-ray emitters. The so-called weak line T Tauri stars (WTTSs) do not appear to experience disk accretion, while the classical T Tauri stars (CTTSs) do. CTTSs exhibit broad emission lines and sometimes also forbidden emission lines. They are spectroscopically and photometrically variable, and have ultraviolet (UV), optical, and infrared (IR) excesses with respect to the photospheric flux.

Magnetospheric accretion models provide the closest description of the main observed characteristics of CTTSs (Shu et al. 1994; Hartmann et al. 1994; Muzerolle et al. 2001;

^ The CoRoT space mission was developed and is operated by the French space agency CNES, with participation of ESA's RSSD and Science Programmes, Austria, Belgium, Brazil, Germany, and Spain.

$\star \star$ Table 1 is only available in electronic form at http: //www. aanda.org
Kurosawa et al. 2006). In these models, the stellar magnetosphere is strong enough to disrupt the circumstellar disk at a few stellar radii. Material in the inner disk, ionized by stellar radiation, falls towards the star following magnetic field lines and hits the stellar photosphere at near free-fall velocities, creating hot spots on the stellar surface. Part of the ionized material in the inner disk region is ejected by a magnetically controlled wind. In this scenario, the broad permitted emission lines are formed partly in the accretion funnel and the hot spot emits a continuum flux that is responsible for the UV and optical excess that veils the photospheric lines. The IR excess comes from the reprocessing by the dust in the disk of the stellar and accretion radiation and, at least for high accretion-rate systems, possibly also viscous heating. When observed, forbidden emission lines are understood to be formed in the low density wind.

In the past decade, numerical simulations of accreting young stars have predicted that there is a very dynamical star-disk interaction, mediated by the stellar magnetic field (Goodson et al. 1999; Matt et al. 2002; Romanova et al. 2009; Zanni 2009). 
Many magneto-hydrodynamical (MHD) model predictions are derived from the idea that the stellar magnetic field interacts with the inner disk region near the corotation radius but not only at the corotation point. Because of the differential rotation between the star and the inner disk region, the magnetic field lines become distorted after a few rotational periods and eventually reconnect, restoring the initial field configuration. This process continues while the star rotates. The timescale of the reconnection events depends on the diffusivity of the stellar magnetic field lines in the inner disk region, which is a very poorly constrained parameter.

AA Tau is one of few CTTSs studied in sufficient detail to test the MHD model dynamical predictions. The star was observed photometrically for a month during three different campaigns, two of which included high-resolution simultaneous spectroscopy (Bouvier et al. 1995, 2003, 2007). AA Tau exhibits a light curve (LC) with a flat maximum interrupted by deep quasi-periodical minima that vary in depth and width from one rotational cycle to the other. The minima occur with little color change and are thought to be due to obscuration of the stellar photosphere by circumstellar disk material present in an inner disk warp. The warp is caused by the interaction of the stellar magnetic field, inclined with respect to the rotation axis, and the inner disk region. The spectroscopic results showed that the magnetic field lines are inflated in the course of a few rotational cycles, as measured by the radial velocity of the red and blue absorption components of the $\mathrm{H} \alpha$ line. When the field lines are most inflated, accretion onto the star is suppressed, with no apparent veiling, and low emission-line equivalent widths, which again corroborates the MHD model predictions. After reconnection, accretion begins all over again. The last two AA Tau observational campaigns were separated by 5 years and the field line inflation discovered in the second campaign was still present with the same characteristics in the third one.

Although the AA Tau observations provided strong support to the MHD results, it has remained unclear whether this behaviour was common among CTTSs. To test this, one required good photometric measurements of a large number of CTTSs, covering several rotational cycles. In Taurus, the typical rotational period of a CTTS is around 8 days, so that stars need to be monitored for at least a month continuously. This is not an easy task from the ground, because of telescope time allocation and weather conditions. The CoRoT satellite additional program to observe the young stellar cluster NGC 2264 has allowed us to perform this study on a large sample of CTTSs.

NGC 2264 is a well-studied young stellar cluster with an age of $\approx 3$ Myr located at a distance of about 760 pc (see Dahm 2008, for a review of the cluster). We matched the various available datasets on the cluster from the literature based on spatial coincidence using a $2^{\prime \prime}$ tolerance. In a small number of cases, double identifications (more than one object within $2^{\prime \prime}$ ) were transformed into single ones, especially in cases where one of the two objects had a small offset and the other a large one relative to the coordinates listed in the various datasets. Owing to the numerous studies of the cluster, from UV to X-rays, we were able to establish reasonable criteria for cluster membership. As likely members of NGC 2264, we considered stars selected according to one or more of the following criteria: i) photometric $\mathrm{H} \alpha$ and variability from the data of Lamm et al. (2004) and following their criteria; ii) X-ray detection (Ramírez et al. 2004; Flaccomio et al. 2006) and location on the cluster sequence in the $(I, R-I)$ diagram if $R$ and $I$ magnitudes are available; iii) spectroscopic $\mathrm{H} \alpha$ equivalent width greater than $10 \AA$; and iv) $\mathrm{H} \alpha$ emission line width at $10 \%$ intensity greater than $270 \mathrm{~km} \mathrm{~s}^{-1}$, as proposed by
White \& Basri (2003) to identify accreting T Tauri stars, and measured by Fưrész et al. (2006) for many cluster members.

The mean rotational period of CTTSs in NGC 2264 is around 3 to 4 days (see Sect. 4). CoRoT observed the cluster for 23 days uninterruptedly, covering several rotational cycles for most of the stars, thus allowing the identification of AA Taulike candidates and the determination of precise rotational periods for cluster members. The complete rotation analysis will be discussed separately in another paper (Affer et al., in prep.).

\section{Observations}

CoRoT observed NGC 2264 from 2008 March 7 to 30. The whole cluster fitted into one of the two CCDs normally used for the exo-planet observations, and stars were observed down to $R \simeq 18$. We used the light curves delivered by the CoRoT pipeline after nominal corrections (Samadi et al. 2006). We corrected the pipeline light curves by removing outliers, related mainly to South Atlantic Anomaly crossings, by applying a sigma-clipping filter, taking care not to remove flaring events. The data were also corrected for the effects of the entrance and exit into Earth eclipses. We did not use the color information provided by CoRoT for the brightest stars, and based our analysis on the broadband, "white light" light curves. All the light curves presented here were rebinned to $512 \mathrm{~s}$ and correspond to the integrated flux in the CoRoT mask. The flux RMS over $512 \mathrm{~s}$ achieved is of the order of 0.0005 for a $R=12$ star and 0.004 for a $R=16$ star, representing the most detailed light curves of young accreting systems yet obtained, as shown in Fig. 1. A complete catalog of the observations will be published in a future paper (Favata et al. in preparation).

\section{Results}

\subsection{Classical T Tauri sample selection}

After selecting the cluster members as described in Sect. 1, we classified stars as CTTSs if either their $\mathrm{H} \alpha$ equivalent width was greater than $10 \AA$, their $U-V$ excess smaller than a threshold calculated below, or their $\mathrm{H} \alpha$ width at $10 \%$ intensity was greater than $270 \mathrm{~km} \mathrm{~s}^{-1}$. Some stars had more than one of the above-mentioned characteristics. White \& Basri (2003) demonstrated that the accretion criterium based on $\mathrm{H} \alpha$ equivalent width is spectral-type dependent, the threshold being smaller than $10 \AA$ for spectral types earlier than K7 and greater than $10 \AA$ for spectral types later than M2.5. Whenever spectral type information was available, we followed the accretion criteria proposed by White \& Basri (2003), instead of using the standard $10 \AA$ value. Rebull et al. (2002) proposed that stars in NGC 2264 with a $U-V$ excess smaller than -0.5 be classified as disk candidates. This threshold value was based on a study of the Orion starforming region (Rebull et al. 2000). However, the $U-V$ excess is also expected to be spectral-type dependent, stars with later spectral types having larger $U-V$ excess than earlier ones, for the same mass accretion rate, because of the greater contrast between the hot spot and the stellar photosphere in later spectral types. Therefore we searched for all stars in our sample that had been selected as CTTS based on both their $U-V$ excess and another criterion ( $\mathrm{H} \alpha$ equivalent width or $\mathrm{H} \alpha$ width at $10 \%$ intensity). We separated them into two spectral type ranges (K0-K6 with 14 stars, and K7-M3 with 10 stars) and computed the mean $U-V$ excesses of each dataset. The K0-K6 CTTSs have a typical $U-V$ excess of $-1.06 \pm 0.48 \mathrm{mag}$ and the K7-M3 CTTSs 

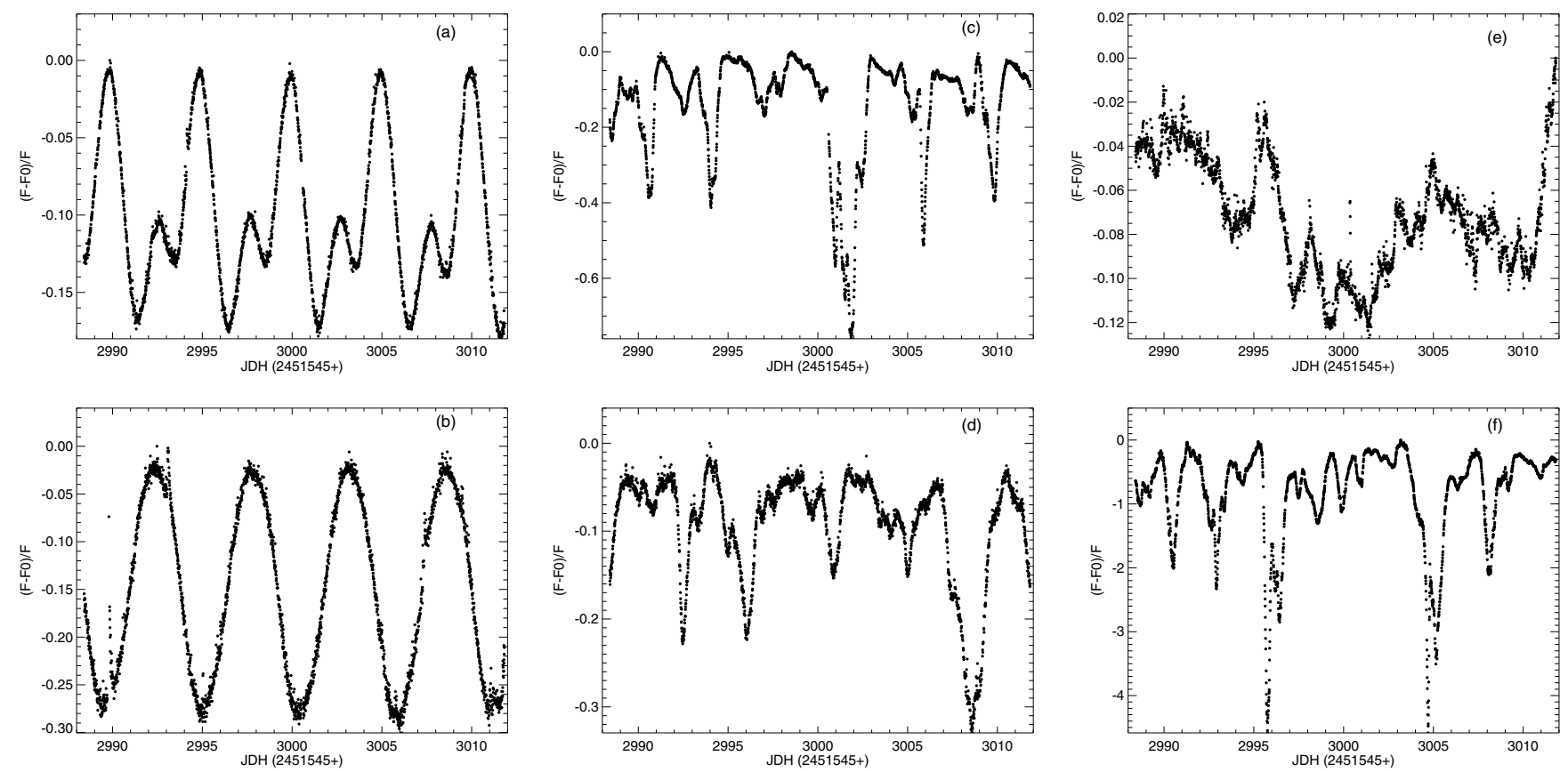

Fig. 1. A sample of 6 CTTS light curves from the COROT observation of NGC 2264. $F_{0}$ corresponds to the maximum flux value of each LC. Light curves $a$ and $b$ have been classified as spot-like, $c$ and $d$ as AA Tau-like, and $e$ and $f$ as irregular.

a typical $U-V$ excess of $-1.69 \pm 0.57 \mathrm{mag}$. Using the one $\sigma$ upper boundary in each spectral type range as a threshold for differentiating CTTS from WTTS $(-0.58$ for K0-K6, and -1.12 for K7-M3), we selected 7 stars as CTTSs based only on their $U-V$ excess. While the value of -0.5 mag proposed by Rebull et al. (2002) seems to be adequate for stars in the K0-K6 spectral range, it is apparently too large for selecting K7-M3 CTTSs in NGC 2264.

We found 83 CTTSs among the 301 observed cluster members and we present in Table 1 the data used to make the CTTS classification. The $\mathrm{H} \alpha$ equivalent width was taken from Rebull et al. (2002) and Dahm \& Simon (2005), except for six stars (CID 223957455, 223959618, 223964667, 223968688, 223991832,223994721 ) for which we measured the $\mathrm{H} \alpha$ equivalent width ourselves, using high resolution hectoechelle spectra kindly provided by Gabor Fürész. The $U-V$ excess data were obtained from Rebull et al. (2002) and Fallscheer \& Herbst (2006) (a data table was kindly provided by Cassandra Fallscheer), and the $\mathrm{H} \alpha$ width at $10 \%$ intensity was taken from Fúrész et al. (2006).

Some CTTSs selected based on their $U-V$ excess had $\mathrm{H} \alpha$ values smaller than $10 \AA$ and would therefore not be selected as CTTS based only on their $\mathrm{H} \alpha$ equivalent width. However, their $U-V$ excess values are smaller than our established threshold values and in the same range as many other systems with either $\mathrm{H} \alpha$ equivalent widths greater than $10 \AA$ or $\mathrm{H} \alpha$ widths at $10 \%$ larger than $270 \mathrm{~km} \mathrm{~s}^{-1}$. We took into consideration that both $\mathrm{H} \alpha$ equivalent width and $U-V$ excess are strongly variable in these stars and were not measured simultaneously. It therefore appears reasonable to use both criteria to select possible CTTSs.

\subsection{Morphological light curve classification}

We searched for periodical variations in the LCs of the observed CTTSs, using the Scargle periodogram as modified by Horne \& Baliunas (1986), and found that 51 of 83 CTTSs exhibited periodical variability. Periodic LCs were divided into two groups: group PI, containing sinusoidal-like LCs with a stable shape from cycle to cycle, and group PII, flat-topped LCs with a clear maximum interrupted by minima that varied in width and depth from cycle to cycle. The variations in group PI are associated with long-lived spots with lifetimes of at least of weeks, while group PII is associated with AA Tau-like systems, where most of the variability is caused by the obscuration of the stellar photosphere by circumstellar disk material. The nonperiodical LCs (group NP) may be due to either obscuration by non-uniformly distributed circumstellar material or non-steady accretion, or both.

A total of 83 CTTSs belonging to NGC 2264 were observed by CoRoT, 28 of which were classified as spot-like (group PI), 23 as AA Tau-like (group PII), and 32 as irregular (group NP). A sample of light curves of each type is shown in Fig. 1. In Fig. 2, we present the periodical LCs of Fig. 1 folded in phase with the periods determined with the Scargle periodogram as modified by Horne \& Baliunas (1986). We note the stability of the spot-like LCs (Figs. 1 and 2a and b) on the timescale of the observations, which makes them, in general, easily distinguishable from the AA Tau-like ones (Figs. 1 and $2 c$ and d). Among the irregular LCs, some more closely resemble variable accretion events (variable hot spots, Fig. 1e) and others obscuration by non-uniform circumstellar material (Fig. 1f), but it is difficult to decide which process is the dominant one based only on the CoRoT light curves without any color information. Therefore we did not make any additional attempt to classify the irregular systems.

We measured the variability amplitude of a LC as ((Flux $\max _{\text {- }}$ Flux $\left._{\min }\right) /$ Flux $\left._{\text {median }}\right) \times 100$. In our sample, the observed CTTS variability amplitudes range from $3 \%$ to $137 \%$, excluding flaring events. The variability amplitude of spot-like LCs is generally between about $10 \%$ and $15 \%$ and most of the stars that have a variability amplitude larger than $20 \%$ have LCs most likely produced by the obscuration of the stellar photosphere by circumstellar material (AA Tau-like). The AA Tau system variability amplitude, measured from data in the literature, is $76 \%$ and 8 of 

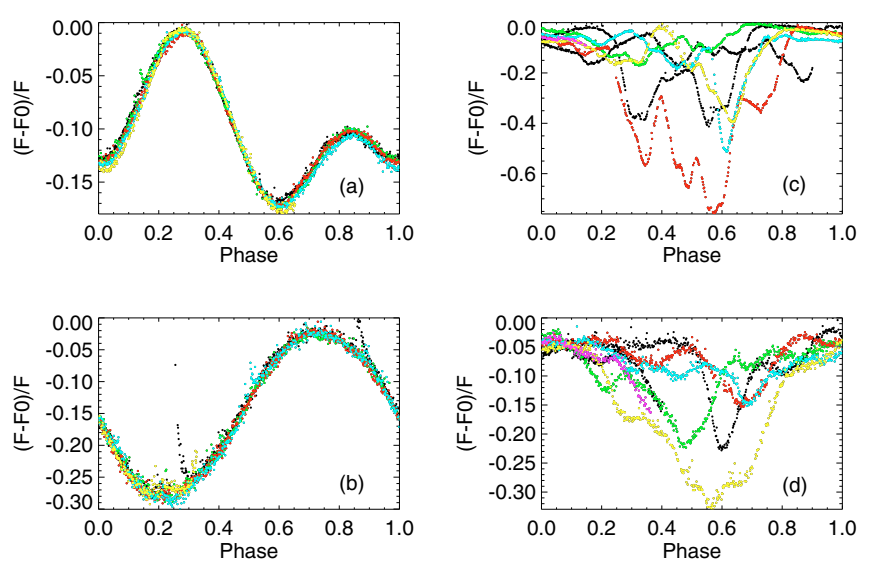

Fig. 2. The four periodic LCs from Fig. 1 folded in phase. Different colors correspond to different cycles.

83 stars observed by CoRoT have a higher variability amplitude than AA Tau. Although AA Tau has a high variability amplitude, probably because of its high inclination to our line of sight $\left(\sim 75^{\circ}\right.$ Bouvier et al. 2003), it is not exceptional among CTTS systems.

From the results in this section, it is clear that the AA Tau photometric behavior is not an exception, but a rather common occurrence among young stellar systems, that is typical of $28 \% \pm 6 \%$ of the CTTS systems in NGC 2264 observed with CoRoT. The percentage of AA Tau-like systems among the observed CTTSs seems reasonable, given that only some geometric configurations (high inclination) would enable the occultation of the observed stellar photosphere, and the chances of occultation events also depend on the disk warp's location and scale-height. This result is however higher than the value of $10 \%$ to $15 \%$ calculated by Bertout (2000) using flared disk models to estimate the probability of observing partial occultation events in CTTS systems. However, the disk models of Bertout (2000) do not include an inner disk warp, and consequently underestimate the probability of obscuration. Assuming a random distribution of axial inclinations, the fraction of AA Tau-like LCs in our sample $(\geq 28 \%)$ implies that $h / r \sim 0.3$ for inner disk warps, where $h$ and $r$ are the inner warp height and distance to the central star. This is larger than the standard value used in $\alpha$-disk models, where $h / r \sim 0.05-0.1$ (Bertout et al. 1988; Duchêne et al. 2010).

\subsection{Merging CoRoT and Spitzer data}

Spitzer IRAC data were also available for the cluster (Teixeira 2008), a total of 68 CTTSs having been observed by both Spitzer and CoRoT. IRAC is useful for identifying near-infrared excess emission originating in warm dusty circumstellar material. We used the $\alpha_{\text {IRAC }}$ index, which represents the slope of the spectral energy distribution between $3.6 \mu \mathrm{m}$ and $8 \mu \mathrm{m}$, to classify the inner disk structure of the observed systems, following the criteria proposed by Lada et al. (2006). Stars with $\alpha_{\text {IRAC }}<-2.56$ are classified as naked photospheres (i.e., these systems are devoid of dust within a few AU), stars with $-2.56<\alpha_{\text {IRAC }}<$ -1.80 have anemic disks (optically thin inner disks), stars with $-1.80<\alpha_{\text {IRAC }}<-0.5$ have optically thick inner disks (referred to as thick disks henceforth), those with $-0.5<\alpha_{\text {IRAC }}<0.5$ are flat spectra sources, and those with $\alpha_{\text {IRAC }}>0.5$ are Class I objects.

We combined both datasets in order to ascertain whether the light curve morphology was related to the evolution of the inner disk structure. The result, presented in Fig. 3, shows that the agreement between the two classification methods for CTTS (based on the CoRoT light curves and on the Spitzer photometry) is excellent. None of the systems with naked photospheres exhibit AA Tau-like LCs and 10/11 $(91 \% \pm 29 \%)$ of them show pure spot-like variability. The percentage of AA Tau-like LCs, which are due to obscuration by circumstellar disk material, thus increases from $0 \%$ for the evolved inner disk systems (naked photospheres) to $36 \% \pm 16 \%$ for the anemic disk systems and $40 \% \pm 10 \%$ for the thick disk systems. Because some of the nonperiodic LCs may also be partly caused by circumstellar obscuration (see a possible example in Fig. 1f), the fractional estimates of obscuration LCs are lower limits for systems with anemic and thick disks.

Spot-like systems represent $91 \% \pm 29 \%$ of the naked photospheres, $28 \% \pm 14 \%$ of the anemic disks and $18 \% \pm 7 \%$ of thick disk systems. They are observed in systems where there is no obscuring material along our line of sight towards the star. This may be caused by inner disk clearing, which may explain the spot-like LCs of naked photosphere systems and some anemic disks, shown in Fig. 3. Spot-like LCs may also originate in low inclination systems. In this case, there may be inner disk material, but this would not obscure the star. This probably corresponds to some anemic disk systems and to all thick disk systems exhibiting spot-like LCs.

The non-periodical LCs represent $9 \% \pm 9 \%$ of the naked photospheres, $36 \% \pm 16 \%$ of the anemic disks, and $42 \% \pm 10 \%$ of the thick disk systems. The irregular LCs among naked photosphere systems may be due to non-steady accretion, which can produce short-lived and variable hot spots. Some irregular systems among anemic disks and thick disks are also probably because of a combination of non-steady accretion and low inclination, as apparently observed in TW Hya (Rucinski et al. 2008). It may however not be straightforward to assign non-periodical LCs to non-steady accretion, as shown by Kulkarni \& Romanova (2009). They computed MHD models of the interaction between a magnetized star and its circumstellar disk with non-steady accretion and showed that, at large misalignment angles $\left(\theta>25^{\circ}\right)$ between the stellar rotation and magnetic axis, hotspots are approximately fixed on the star's surface, even during strongly unstable accretion, and consequently the LCs always have the stellar rotation period. Assuming that the circumstellar environment of a CTTS is complex, random accretion events due to circumstellar blobs, which fall towards the star, can also temporarily occult the star and explain some of the observed non-periodical systems, as proposed by DeWarf et al. (2003) to explain the irregular photometric variability of the CTTS SU Aur. Another situation that could produce irregular LCs is a flared disk seen at high inclination. We could then expect to see partial obscuration by circumstellar disk material from the disk outer layers. Bertout (2000) calculated the partial obscuration probability for a flared disk with no inner disk warp and showed that, for a typical CTTS, it would be between $10 \%$ and $15 \%$. In this case, assuming Keplerian disk rotation and a typical CTTS disk with an outer radius of $100 \mathrm{AU}$, we would unfortunately not be able to measure short-scale variability, as observed with CoRoT, due to material located at most disk radii because of the limited duration of our observations.

\section{Discussion}

We calculated periods for all CTTSs with periodic variations (51 of 83 CTTSs), using the Scargle periodogram modified by Horne \& Baliunas (1986). We present in Fig. 4 the period distribution of the spot-like (horizontally hatched histogram) and AA Tau-like 
Sample of NGC 2264 CTTSs

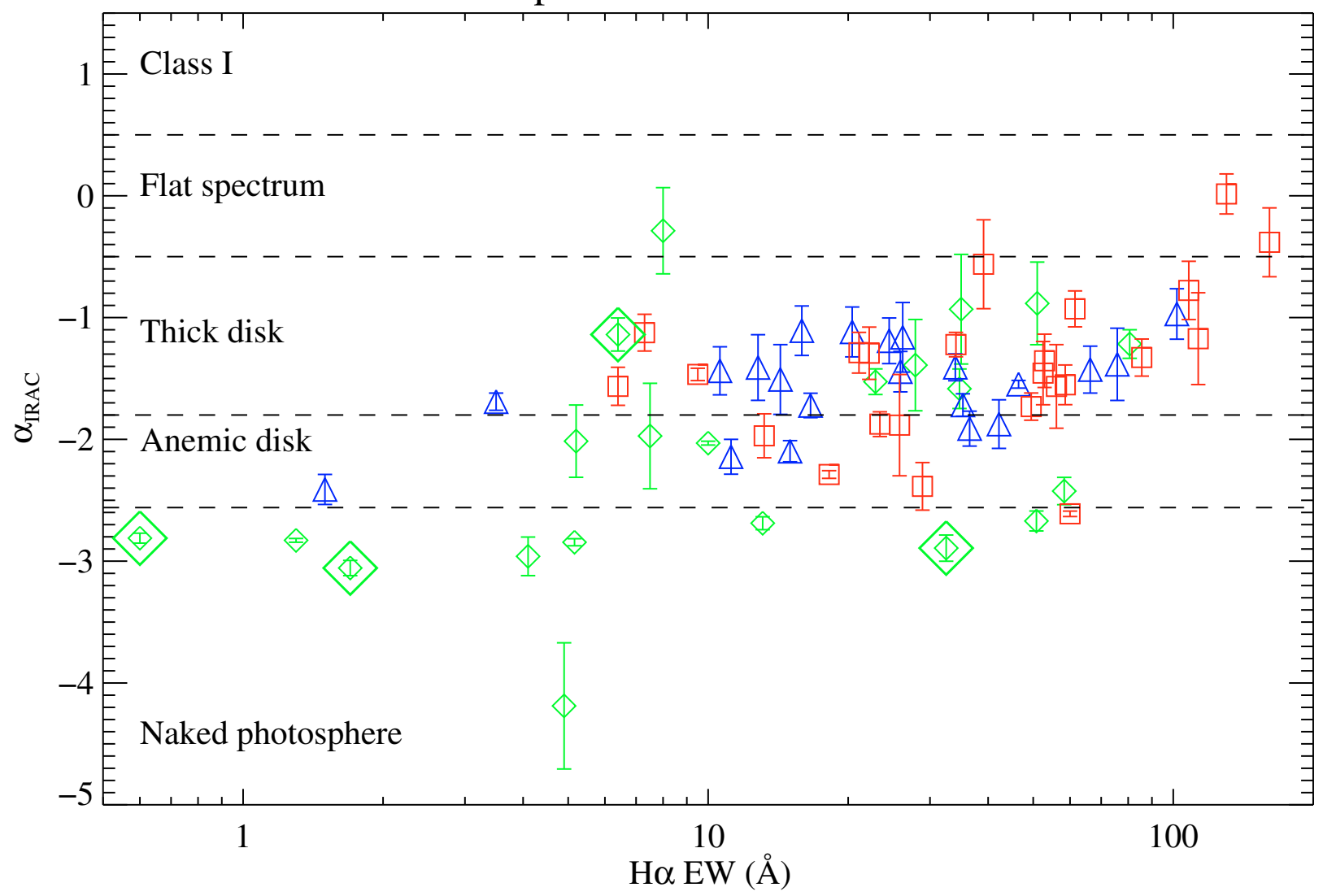

Fig. 3. NGC 2264 classical T Tauri stars observed with CoRoT and Spitzer. Diamonds correspond to spot-like LCs, triangles to AA Tau-like LCs, and squares to non-periodical LCs. The four stars with large overplotted diamonds are rapid rotators ( $p<2$ days). One rapid rotator was not

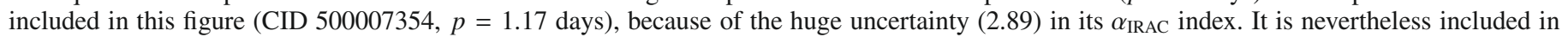
Table 1 and in our LC analysis.

(diagonally hatched histogram) systems. A complete discussion of the period distribution in NGC 2264 will be presented in another paper (Affer et al., in prep.).

The major difference between the period distributions of spot-like and AA Tau-like systems is that rapid rotators $(p<$ 2 days) are only found to have spot-like LCs. Among the seven rapid rotating CTTSs, five also have Spitzer IRAC measurements. Four of them are classified as naked photosphere systems and another is a thick disk system. The thick disk system (CID 223980447, $\alpha_{\text {IRAC }}=-1.14 \pm 0.13, p=1.67$ days) is a K6 star (Dahm \& Simon 2005) with high-resolution hectoechelle spectra (Fúrész et al. 2006) that Gabor Fúrész kindly made available to us. We measured $v \sin i=30 \mathrm{~km} \mathrm{~s}^{-1}$, using the SYNTH3 code provided by Dr. Oleg Kochukhov (Kochukhov 2007), and both MARCS atmospheres (Gustafsson et al. 2008) and atomic lines from VALD (Kupka et al. 2000, 1999). Assuming $R_{*}=$ $2 R_{\odot}$, we obtained $i=29.4^{\circ}$. This low inclination can explain the presence of circumstellar material without any obscuration occurring in the LC. Except for this system, most rapid rotators are found among systems that have cleared their inner disk regions, which may be an indication that as the star-disk coupling decreases, stars tend to spin-up, as also found by Rebull et al. (2006) and Cieza \& Baliber (2007) in Orion and NGC 2264. This is not a straightforward conclusion, however, from our data, since the number of rapid rotators is small and on the other hand some naked photosphere and anemic disk systems are found to rotate slowly, with periods between 10 and 15 days.

The periods measured in the AA Tau-like LCs are in the range of periods obtained from spot-like LCs. The periods measured from the spot-like LCs correspond to stellar rotational periods, since spots are located at the stellar photosphere. This indicates that AA Tau-like periods are within the range of stellar rotational periods of CTTSs in NGC 2264 and therefore the material that obscures the star must be located close to the corotation radius. Since the inner warp is by definition located close to or at the dust disk truncation radius, this implies that this radius is close to the corotation radius in the systems we classified as AA Tau-like. Carr (2007) showed that the inner gas radius is on average slightly smaller than the corotation radius, while the inner dust radius falls at or outside the corotation radius. This is quite consistent with the CoRoT results.

Since the inner disk warp is located near the corotation radius, the variations observed from cycle to cycle in width and depth of the photometric minima should be related to the dynamical star-disk interaction in the inner disk region, which is understood to be responsible for the accumulation of material near the disk truncation region, forming inner disk warps. Like AA Tau, the star-disk interaction is seen to be very dynamic on a rotational timescale, as predicted by MHD models of young magnetized star-disk systems (Goodson et al. 1999; 


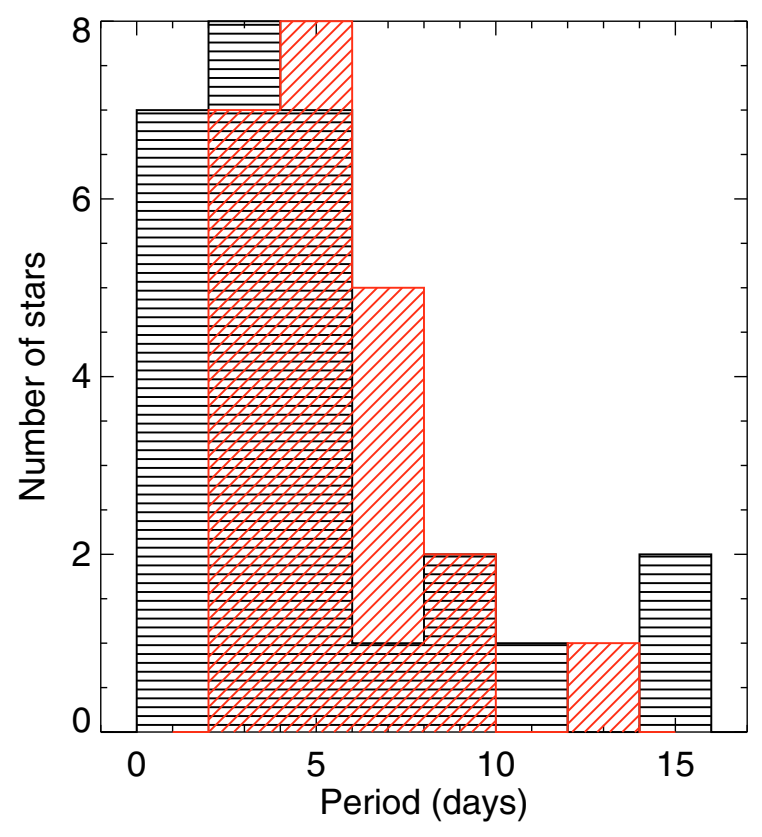

Fig. 4. Period distribution of CTTSs. The horizontally hatched histogram corresponds to spot-like LCs and the diagonally hatched histogram to the AA Tau-like LCs.

Matt et al. 2002; Romanova et al. 2009; Zanni 2009). In our observations, some systems are more regular and stable than others, but it is quite common to discover systems that have photometric minima that vary substantially from cycle to cycle, yet maintain their overall periodic nature (see Figs. 1, 2c and d).

\section{Conclusions}

We have demonstrated that the AA Tau photometric behavior is common among CTTSs, being present in $28 \% \pm 6 \%$ of the CTTSs in our sample. This represents a lower limit, since the AA Tau-like LCs are more likely to be produced at high inclinations, and we have probably missed about $20 \%$ to $30 \%$ of the very high inclination systems, according to the calculations of Bertout (2000), which are totally obscured by a flared disk and thus too faint to be observed by CoRoT.

If our interpretation of these systems is correct, the photometric minima are due to obscuring material located in the inner disk region, near the corotation radius. This material is accumulated by means of the interaction between an inclined stellar magnetosphere and the inner disk region. The observed periodical changes in width and depth of the observed minima, over a timescale of a few $(\sim 1-3)$ rotational periods, indeed reflect the dynamics of this interaction, which is predicted by MHD models of young low mass star-disk systems.

We compared the CoRoT light curves with Spitzer IRAC data of the same systems and showed that the agreement between different classifications based on the two datasets is excellent. The percentage of AA Tau-like light curves, which are produced by the obscuration of the stellar photosphere by circumstellar material in the inner disk region, varies as the inner disk dissipates, decreasing from $40 \% \pm 10 \%$ in systems with thick inner disks to $36 \% \pm 16 \%$ in systems with anemic disks and zero in naked photosphere systems. Indeed, $91 \% \pm 29 \%$ of the systems with naked photospheres exhibit pure spot-like variability, while only $18 \% \pm 7 \%$ of the thick disk systems do so, presumably those seen at low inclination and thus free of variable obscuration.

Acknowledgements. We thank Gabor Fưrész for making his Hectoechelle spectra and the updated electronic version of his tables available to us. We thank Cassandra Fallscheer for making available to us her table of $U-V$ excess measurements. This research is based on data collected on the CoRoT satellite. SHPA and MMG acknowledge support from CNPq, CAPES and Fapemig. JFG acknowleges support from the FCT project PTDC/CTE-AST/66181/2006.

\section{References}

Bertout, C. 2000, A\&A, 363, 984

Bertout, C., Basri, G., \& Bouvier, J. 1988, ApJ, 330, 350

Bouvier, J., Covino, E., Kovo, O., et al. 1995, A\&A, 299, 89

Bouvier, J., Grankin, K. N., Alencar, S. H. P., et al. 2003, A\&A, 409, 169

Bouvier, J., Alencar, S. H. P., Boutelier, T., et al. 2007, A\&A, 463, 1017

Carr, J. S. 2007, IAU Symp., 243, 135

Cieza, L., \& Baliber, N. 2007, ApJ, 671, 605

Dahm, S. E. 2008, Handbook of Star Forming Regions, Volume I: The Northern

Sky ASP Monograph Publications, ed. Bo Reipurth, 4, 966

Dahm, S. E., \& Simon, T. 2005, AJ, 129, 829

DeWarf, L. E., Sepinsky, J. F., Guinan, E. F., Ribas, I., \& Nadalin, I. 2003, ApJ, 590,357

Duchêne, G., McCabe, C., Pinte, C., et al. 2010, ApJ, 712, 112

Fallscheer, C., \& Herbst, W. 2006, ApJ, 647, L155

Flaccomio, E., Micela, G., \& Sciortino, S. 2006, A\&A, 455, 903

Fûrész, G., Hartmann, L. W., Szentgyorgyi, A. H., et al. 2006, ApJ, 648, 1090

Goodson, A. P., Böhm, K.-H., \& Winglee, R. M. 1999, ApJ, 524, 142

Gustafsson, B., Edvardsson, B., Eriksson, K., et al. 2008, A\&A, 486, 951

Hartmann, L., Hewett, R., \& Calvet, N. 1994, ApJ, 426, 669

Horne, J. H., \& Baliunas, S. L. 1986, ApJ, 302, 757

Kochukhov, O. 2007, Physics of Magnetic Stars, ed. I. I. Romanyuk, \& D. O. Kudryavtsev, Proc. Int. Conf., 109

Kulkarni, A. K., \& Romanova, M. M. 2009, MNRAS, 398, 701

Kupka, F., Piskunov, N. E., Ryabchikova, T. A., Stempels, H. C., \& Weiss, W. W. 1999, A\&AS, 138, 119

Kupka, F., Ryabchikova, T. A., Piskunov, N. E., Stempels, H. C., \& Weiss, W. W. 2000, Baltic Astron., 9, 590

Kurosawa, R., Harries, T. J., \& Symington, N. H. 2006, MNRAS, 370, 580

Lada, C. J., Muench, A. A., Luhman, K. L., et al. 2006, AJ, 131, 1574

Lamm, M. H., Bailer-Jones, C. A. L., Mundt, R., Herbst, W., \& Scholz, A. 2004, A\&A, 417, 557

Matt, S., Goodson, A. P., Winglee, R. M., \& Böhm, K.-H. 2002, ApJ, 574, 232

Ménard, F., \& Bertout, C. 1999, NATO ASIC Proc. 540: The Origin of Stars and Planetary Systems, 341

Muzerolle, J., Hartmann, L., \& Calvet, N. 1998, AJ, 116, 455

Muzerolle, J., Hartmann, L., \& Calvet, N. 2001, ApJ, 550, 944

Ramírez, S. V., Rebull, L., Stauffer, J., et al. 2004, AJ, 127, 2659

Rebull, L. M., Hillenbrand, L. A., Strom, S. E., et al. 2000, AJ, 119, 3026

Rebull, L. M., Makidon, R. B., Strom, S. E., et al. 2002, AJ, 123, 1528

Rebull, L. M., Stauffer, J. R., Megeath, S. T., Hora, J. L., \& Hartmann, L. 2006, ApJ, 646, 297

Romanova, M. M., Ustyugova, G. V., Koldoba, A. V., \& Lovelace, R. V. E. 2009, MNRAS, accepted [arXiv: 0907.3394]

Rucinski, S. M., Matthews, J. M., Kuschnig, R., et al. 2008, MNRAS, 391, 1913 Samadi, R., Fialho, F., Costa, J. E. S., et al. 2006, ESA SP 1306, 317, corrected in [arXiv: astro-ph/0703354]

Scargle, J. D. 1982, ApJ, 263, 835

Shu, F., Najita, J., Ostriker, E., et al. 1994, ApJ, 429, 781

Teixeira, P. S. 2008, Ph.D. Thesis

White, R. J., \& Basri, G. 2003, ApJ, 582, 1109

Zanni, C. 2009, Lecture Notes in Physics (Berlin: Springer Verlag), 791, 155

Pages 7 to 8 are available in the electronic edition of the journal at http: //www . aanda. org 
Table 1. Classical T Tauri stars that belong to NGC 2264 and were observed by CoRoT.

\begin{tabular}{|c|c|c|c|c|c|c|c|c|c|c|c|}
\hline $\begin{array}{l}\text { RA } \\
\text { (deg) }\end{array}$ & $\begin{array}{l}\text { Dec } \\
(\mathrm{deg})\end{array}$ & $\begin{array}{l}V \\
\text { (mag) }\end{array}$ & CoRoT ID & $\begin{array}{l}\mathrm{H} \alpha E W \\
(\AA)\end{array}$ & $\begin{array}{l}\text { UV1 } \\
\text { (mag) }\end{array}$ & $\begin{array}{l}\text { UV2 } \\
\text { (mag) }\end{array}$ & $\mathrm{H} \alpha 10 \%$ & $\mathrm{SpT}$ & LC group & $\begin{array}{l}\text { Period } \\
\text { (days) }\end{array}$ & $\alpha_{\text {IRAC }}$ \\
\hline 99.89338 & 9.91424 & 16.64 & 223957455 & 22.1 & $\ldots$ & $\ldots$ & $\mathrm{x}$ & 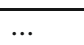 & $\mathrm{NP}$ & & $\ldots$ \\
\hline 99.92281 & 9.77214 & 14.64 & 223959618 & 28.4 & $\ldots$ & $\ldots$ & $\mathrm{x}$ & $\ldots$ & PII & 3.87 & $\ldots$ \\
\hline 99.99684 & 9.45681 & 16.34 & 223964667 & 42.2 & $\ldots$ & $\ldots$ & $\mathrm{x}$ & $\ldots$ & PII & 6.45 & -1.87 \\
\hline 100.04666 & 9.63501 & 16.67 & 223968039 & 52.9 & -1.15 & $\ldots$ & $\mathrm{x}$ & K6 & NP & $\ldots$ & -1.35 \\
\hline 100.05673 & 9.41375 & 13.02 & 223968688 & 0.60 & $\ldots$ & $\ldots$ & $\mathrm{x}$ & K0 & PI & 1.11 & -2.81 \\
\hline 100.05710 & 9.94183 & 17.02 & 400007614 & 130.2 & $\ldots$ & $\ldots$ & $\mathrm{x}$ & M2 & NP & $\ldots$ & 0.0149 \\
\hline 100.09620 & 9.46176 & 16.04 & 223971231 & 49.5 & -1.62 & $\ldots$ & $\mathrm{x}$ & K5 & NP & $\ldots$ & -1.73 \\
\hline 100.09889 & 9.92330 & 17.55 & 223971383 & 80.6 & $\ldots$ & $\ldots$ & $\mathrm{x}$ & $\ldots$ & PI & 4.66 & -1.22 \\
\hline 100.12006 & 9.51718 & 15.20 & 223972652 & 39.1 & -0.89 & $\ldots$ & $\ldots$ & K4 & NP & $\ldots$ & -0.56 \\
\hline 100.12186 & 9.73542 & 17.69 & 400007809 & 31.3 & -1.36 & $\ldots$ & $\mathrm{x}$ & M2 & NP & $\ldots$ & $\ldots$ \\
\hline 100.12859 & 9.57794 & 16.03 & 223973200 & 22.2 & $\ldots$ & $\ldots$ & $\mathrm{x}$ & K1 & NP & $\ldots$ & -1.29 \\
\hline 100.13013 & 9.51864 & 15.43 & 223973292 & 1.7 & -0.91 & $\ldots$ & $\ldots$ & K6 & PI & 1.96 & $\ldots$ \\
\hline 100.15217 & 9.84601 & 16.67 & 400007538 & 21.1 & -2.11 & -1.687 & $\ldots$ & M2 & NP & $\ldots$ & -1.29 \\
\hline 100.15262 & 9.80638 & 15.62 & 500007298 & 4.9 & -1.43 & -0.606 & $\ldots$ & K5 & PI & 15.70 & -4.19 \\
\hline 100.15781 & 9.58167 & 16.64 & 400007528 & 23.4 & $\ldots$ & $\ldots$ & $\mathrm{x}$ & M3 & NP & $\ldots$ & -1.87 \\
\hline 100.16297 & 9.84961 & 15.43 & 500007252 & 46.5 & -1.89 & $\ldots$ & $\mathrm{x}$ & K4 & PII & 7.06 & -1.55 \\
\hline 100.16840 & 9.84735 & 15.50 & 500007272 & 58.3 & $\ldots$ & $\ldots$ & $\ldots$ & K7 & PI & 3.76 & -2.42 \\
\hline 100.17086 & 9.46509 & 16.53 & 500007505 & 13.2 & $\ldots$ & $\ldots$ & $\ldots$ & $\ldots$ & NP & $\ldots$ & -1.97 \\
\hline 100.17095 & 9.79936 & 16.01 & 500007379 & 7.5 & -1.22 & -0.984 & $\ldots$ & K7 & PI & 14.15 & -1.97 \\
\hline 100.17216 & 9.85066 & 15.70 & 500007315 & 24.5 & -2.13 & $\ldots$ & $\ldots$ & K7 & PII & 7.80 & -1.19 \\
\hline 100.17233 & 9.90385 & 13.57 & 223975844 & 12.2 & 0.02 & $\ldots$ & $\mathrm{x}$ & G3 & PI & 3.31 & $\ldots$ \\
\hline 100.17435 & 9.86237 & 15.49 & 500007269 & 23.4 & $\ldots$ & $\ldots$ & $\ldots$ & K5 & PI & 3.75 & -1.53 \\
\hline 100.17576 & 9.56040 & 12.09 & 223976028 & 7.3 & $\ldots$ & $\ldots$ & $\mathrm{x}$ & G0 & NP & $\ldots$ & -1.12 \\
\hline 100.18006 & 9.78535 & 16.33 & 500007460 & 27.1 & $\ldots$ & $\ldots$ & $\mathrm{x}$ & K6 & NP & $\ldots$ & $\ldots$ \\
\hline 100.18580 & 9.54061 & 18.13 & 500007930 & 60.0 & $\ldots$ & $\ldots$ & $\ldots$ & M3 & NP & $\ldots$ & -2.61 \\
\hline 100.18819 & 9.47901 & 14.55 & 223976747 & 7.2 & -0.16 & $\ldots$ & $\mathrm{x}$ & $\mathrm{K} 2$ & PII & 3.16 & $\ldots$ \\
\hline 100.19793 & 9.82471 & 14.14 & 500007120 & 12.8 & $\ldots$ & -0.806 & $\mathrm{x}$ & K1 & PII & 4.23 & -1.41 \\
\hline 100.19968 & 9.55087 & 18.24 & 500007963 & $\ldots$ & $\ldots$ & -0.917 & $\ldots$ & $\ldots$ & PI & 2.56 & $\ldots$ \\
\hline 100.20505 & 9.96077 & 17.46 & 500007730 & 50.8 & $\ldots$ & $\ldots$ & $\ldots$ & M1 & PI & 11.85 & -2.67 \\
\hline 100.20789 & 9.61375 & 15.37 & 223977953 & 66.3 & -1.79 & -1.906 & $\ldots$ & K4 & PII & 4.96 & -1.43 \\
\hline 100.21081 & 9.91593 & 15.13 & 500007209 & 11.2 & $\ldots$ & $\ldots$ & $\mathrm{x}$ & $\mathrm{K} 2$ & PII & 2.51 & -2.14 \\
\hline 100.21326 & 9.74615 & 12.05 & 223978308 & 3.5 & $\ldots$ & $\ldots$ & $\mathrm{x}$ & G0 & PII & 5.40 & -1.69 \\
\hline 100.22346 & 9.55686 & 14.70 & 223978921 & 18.2 & $\ldots$ & -0.643 & $\mathrm{x}$ & K1 & NP & $\ldots$ & -2.29 \\
\hline 100.22607 & 9.82232 & 16.39 & 500007473 & 161.1 & -2.11 & $\ldots$ & $\mathrm{x}$ & M0 & $\mathrm{NP}$ & $\ldots$ & -0.38 \\
\hline 100.22990 & 9.84718 & 15.89 & 500007354 & 2.8 & -0.64 & -0.774 & $\ldots$ & K5.5 & PI & 1.17 & -9.15 \\
\hline 100.23215 & 9.85385 & 15.54 & 500007283 & 8.00 & $\ldots$ & $\ldots$ & $\ldots$ & K5.5 & PI & 3.23 & -0.29 \\
\hline 100.23663 & 9.63029 & 15.09 & 223979728 & 113.2 & $\ldots$ & -1.353 & $\mathrm{x}$ & M1 & NP & & -1.17 \\
\hline 100.24208 & 9.61483 & 18.05 & 223980048 & 34.0 & $\ldots$ & $\ldots$ & $\ldots$ & $\ldots$ & PII & 4.06 & -1.41 \\
\hline 100.24447 & 9.60368 & 17.40 & 223980233 & 22.2 & $\ldots$ & $\ldots$ & $\ldots$ & M4 & NP & $\ldots$ & $\ldots$ \\
\hline 100.24510 & 9.65522 & 17.02 & 223980258 & 27.9 & $\ldots$ & $\ldots$ & $\mathrm{x}$ & M0 & PI & 7.05 & -1.39 \\
\hline 100.24516 & 9.51592 & 14.04 & 223980264 & 14.3 & $\ldots$ & $\ldots$ & $\mathrm{x}$ & $\mathrm{K} 2.5$ & PII & 3.46 & -1.51 \\
\hline 100.24770 & 9.99596 & 15.34 & 223980412 & 7.41 & -0.53 & $\ldots$ & $\ldots$ & K5 & PI & 3.23 & $\ldots$ \\
\hline 100.24792 & 9.49770 & 17.00 & 500007610 & 26.2 & $\ldots$ & $\ldots$ & $\ldots$ & M3 & PII & 4.66 & -1.16 \\
\hline 100.24811 & 9.58636 & 15.58 & 223980447 & 6.4 & -1.01 & -0.427 & $\mathrm{x}$ & K6 & PI & 1.67 & -1.14 \\
\hline 100.25209 & 9.75088 & 15.39 & 223980688 & 15.0 & -0.59 & -0.723 & $\mathrm{x}$ & K3 & PII & 4.16 & -2.10 \\
\hline 100.25214 & 9.48776 & 14.56 & 223980693 & 16.6 & $\ldots$ & $\ldots$ & $\mathrm{x}$ & K4 & PII & 5.35 & -1.72 \\
\hline 100.25323 & 9.85620 & 14.63 & 500007157 & 1.6 & $\ldots$ & -0.658 & $\ldots$ & K1 & PI & 4.36 & $\ldots$ \\
\hline 100.25408 & 9.54568 & 13.51 & 223980807 & 6.4 & $\ldots$ & $\ldots$ & $\mathrm{x}$ & K1 & NP & $\ldots$ & -1.56 \\
\hline 100.25767 & 9.64475 & 14.70 & 223981023 & 1.5 & $\ldots$ & $\ldots$ & $\mathrm{x}$ & K4 & PII & 7.05 & -2.41 \\
\hline 100.26266 & 9.62660 & 19.17 & 500008211 & 34.1 & $\ldots$ & $\ldots$ & $\ldots$ & M1 & NP & $\ldots$ & -1.22 \\
\hline 100.26503 & 9.50806 & 17.67 & 400007803 & 20.4 & $\ldots$ & $\ldots$ & $\ldots$ & $\ldots$ & PII & 9.75 & -1.12 \\
\hline 100.26789 & 9.41449 & 15.79 & 500007335 & 101.8 & -2.34 & $\ldots$ & $\mathrm{x}$ & M0 & PII & 7.36 & -0.97 \\
\hline 100.26905 & 9.64190 & 17.88 & 500007857 & 108. & $\ldots$ & $\ldots$ & $\ldots$ & M3 & NP & $\ldots$ & -0.78 \\
\hline 100.27071 & 9.84613 & 14.36 & 223981811 & 36.5 & -0.29 & -0.816 & $\mathrm{x}$ & K1 & PII & 3.73 & -1.91 \\
\hline 100.27124 & 9.86239 & 15.39 & 500007248 & 1.7 & -0.75 & -0.570 & $\ldots$ & K5 & PI & 1.88 & -3.06 \\
\hline 100.27583 & 9.60638 & 13.52 & 223982136 & 10.0 & $\ldots$ & $\ldots$ & $\mathrm{x}$ & G3 & PI & 3.01 & -2.03 \\
\hline 100.27595 & 9.41769 & 18.01 & 500007896 & 34.7 & $\ldots$ & $\ldots$ & $\ldots$ & M5 & PI & 9.30 & -1.58 \\
\hline 100.27679 & 9.47745 & 17.30 & 400007686 & 56.1 & $\ldots$ & $\ldots$ & $\mathrm{x}$ & M1.5 & NP & $\ldots$ & -1.57 \\
\hline 100.27808 & 9.57943 & 15.97 & 500007369 & 49.4 & $\ldots$ & $\ldots$ & $\mathrm{x}$ & G6 & NP & $\ldots$ & $\ldots$ \\
\hline 100.28734 & 9.56278 & 17.53 & 500007752 & 51.0 & $\ldots$ & $\ldots$ & $\mathrm{x}$ & M3 & PI & 4.01 & -0.88 \\
\hline 100.29582 & 9.59881 & 17.44 & 500007727 & 61.5 & $\ldots$ & -2.037 & $\mathrm{x}$ & K7 & NP & $\ldots$ & -0.93 \\
\hline 100.30241 & 9.87533 & 14.07 & 500007115 & 35.3 & $\ldots$ & $\ldots$ & $\mathrm{x}$ & G & PII & 2.01 & -1.72 \\
\hline 100.30362 & 9.43746 & 13.76 & 500007089 & 85.6 & $\ldots$ & $\ldots$ & $\mathrm{x}$ & K4 & NP & $\ldots$ & -1.33 \\
\hline 100.31035 & 9.62065 & 17.23 & 500007667 & 4.1 & $\ldots$ & -0.697 & $\ldots$ & $\ldots$ & PI & 5.41 & -2.96 \\
\hline 100.32188 & 9.90899 & 15.64 & 223985009 & 58.3 & -2.40 & $\ldots$ & $\ldots$ & K7 & NP & $\ldots$ & $\ldots$ \\
\hline
\end{tabular}


Table 1. continued.

\begin{tabular}{|c|c|c|c|c|c|c|c|c|c|c|c|}
\hline $\begin{array}{l}\text { RA } \\
\text { (deg) }\end{array}$ & $\begin{array}{l}\text { Dec } \\
\text { (deg) }\end{array}$ & $\begin{array}{l}V \\
(\mathrm{mag}) \\
\end{array}$ & CoRoT ID & $\begin{array}{l}\mathrm{H} \alpha E W \\
(\AA)\end{array}$ & $\begin{array}{l}\text { UV1 } \\
\text { (mag) }\end{array}$ & $\begin{array}{l}\text { UV2 } \\
\text { (mag) }\end{array}$ & $\mathrm{H} \alpha 10 \%$ & SpT & LC group & $\begin{array}{l}\text { Period } \\
\text { (days) }\end{array}$ & $\alpha_{\text {IRAC }}$ \\
\hline 100.32467 & 9.48364 & 18.55 & 500008049 & 231.4 & $\ldots$ & $\ldots$ & $\cdots$ & M2.5 & $\mathrm{NP}$ & & -0.68 \\
\hline 100.32534 & 9.64038 & 18.58 & 500008061 & 32.5 & $\ldots$ & $\ldots$ & $\ldots$ & M3 & PI & 0.98 & -2.89 \\
\hline 100.32613 & 9.56488 & 15.02 & 223985261 & 28.9 & -0.97 & $\ldots$ & $\mathrm{x}$ & K4 & NP & $\ldots$ & -2.39 \\
\hline 100.33752 & 9.56005 & 15.20 & 223985987 & 10.6 & $\ldots$ & $\ldots$ & $\mathrm{x}$ & K6 & PII & 3.31 & -1.44 \\
\hline 100.34851 & 9.78788 & 17.93 & 500007872 & 5.2 & $\ldots$ & -0.767 & $\ldots$ & $\ldots$ & PI & 8.20 & -2.01 \\
\hline 100.35227 & 9.62653 & 17.38 & 400007709 & 8.9 & -0.99 & $\ldots$ & $\ldots$ & M3 & PI & 0.76 & $\ldots$ \\
\hline 100.35677 & 9.57861 & 16.15 & 223987178 & 15.9 & -0.61 & -1.233 & $\ldots$ & M0 & PII & 4.96 & -1.11 \\
\hline 100.36250 & 9.50365 & 17.47 & 400007734 & 25.8 & $\ldots$ & $\ldots$ & $\ldots$ & M1 & NP & & -1.88 \\
\hline 100.37968 & 9.44951 & 14.19 & 500007122 & 25.9 & $\ldots$ & $\ldots$ & $\mathrm{x}$ & $\ldots$ & PII & 12.53 & -1.44 \\
\hline 100.38169 & 9.80912 & 14.63 & 223988742 & 5.16 & -0.08 & -0.409 & $\ldots$ & $\mathrm{K} 2$ & PI & 5.03 & -2.84 \\
\hline 100.38331 & 10.0068 & 15.59 & 223988827 & 13.1 & $\ldots$ & $\ldots$ & $\ldots$ & K5 & PI & 4.78 & -2.69 \\
\hline 100.38543 & 9.63540 & 14.66 & 223988965 & 1.3 & -0.67 & $\ldots$ & $\ldots$ & K6 & PI & 3.23 & -2.83 \\
\hline 100.39397 & 9.60904 & 17.16 & 223989567 & 4.5 & -0.83 & $\ldots$ & $\mathrm{x}$ & M1 & $\mathrm{NP}$ & $\ldots$ & $\ldots$ \\
\hline 100.40536 & 9.75186 & 15.44 & 223990299 & 35.0 & $\ldots$ & -0.826 & $\mathrm{x}$ & K4 & PI & 4.51 & -0.93 \\
\hline 100.41155 & 9.53661 & 15.40 & 500007249 & 58.6 & $\ldots$ & $\ldots$ & $\mathrm{x}$ & K4 & NP & $\ldots$ & -1.55 \\
\hline 100.41564 & 9.67443 & 13.86 & 223990964 & 52.5 & $\ldots$ & -1.061 & $\ldots$ & K4 & NP & $\ldots$ & -1.46 \\
\hline 100.42867 & 9.41900 & 16.65 & 223991832 & 75.8 & $\ldots$ & $\ldots$ & $\mathrm{x}$ & $\ldots$ & PII & 8.40 & -1.38 \\
\hline 100.47104 & 9.96747 & 14.99 & 223994721 & 9.5 & $\ldots$ & $\ldots$ & $\mathrm{x}$ & K7 & NP & $\ldots$ & -1.47 \\
\hline
\end{tabular}

Notes. The H $\alpha$ equivalent widths come from Rebull et al. (2002) and Dahm \& Simon (2005), except for six stars (CID 223957455, 223959618, $223964667,223968688,223991832,223994721)$ for which we measured the $\mathrm{H} \alpha$ equivalent width ourselves, using the high resolution hectoechelle spectra kindly provided by Gabor Fürész. The $U-V$ excesses were taken from Rebull et al. (2002) (UV1) and Fallscheer \& Herbst (2006) (UV2, the $U-V$ excess data table was provided by Cassandra Fallscheer). Stars that have an $\mathrm{H} \alpha$ width at $10 \%$ intensity larger than $270 \mathrm{~km} \mathrm{~s}^{-1}$ are identified by a x sign in column eight, following the classification of Fúrész et al. (2006). The LC group and period values were determined in the present work. The $\alpha_{\mathrm{IRAC}}$ index is described in Sect. 3.3 and comes from Teixeira (2008). 